\title{
Preparation and Structural Characterization of Hydrogel Microspheres
}

\author{
Yukiko Kamiso, Keiji Fujimoto, Haruma Kawaguchi, Yoshiaki Yuguchi,* \\ Hiroshi Urakawa, * and Kanji KaJiwara*,† \\ Faculty of Science \& Technology, Keio University, Hiyoshi, Yokohama 223, Japan \\ * Faculty of Engineering \& Design, Kyoto Institute of Technology, \\ Kyoto, Sakyo-ku, Matsugasaki 606, Japan
}

(Received July 27, 1995)

\begin{abstract}
Monodisperse hydrogel microspheres (microgels) were prepared by precipitation polymerization from acrylamide (AAm), methylenebisacrylamide (MBAAm), and methacrylic acid (MAc) in isopropanol. MAc was found to be essential for the preparation of fine spherical particles. The structural analysis of microgels at each step of conversion suggests that the precipitation polymerization proceeds in two steps of (1) the formation of star-shaped nuclei mainly by crosslinking agents and the coupling of the nuclei to constitute a network structure, and (2) the incorporation of remaining acrylamide onto the microgel surface and the further crosslinking with free double bonds in microgels. The particle size was found to depend on various polymerization conditions, including the monomer composition, the amount of initiator and the total monomer concentration. The internal structure of microgels was observed by small-angle X-ray scattering (SAXS). The scattering profile was analyzed by Fourier-transformation to yield the distance distribution function $p(r)$, and by fitting an assumed scattering function to evaluate the correlation length $\xi$ and the fractal dimension $D$.

KEY WORDS Microsphere / Hydrogel/ Precipitation Polymerization / Small-Angle X-Ray Scattering/
\end{abstract}

A hydrogel microsphere (microgel) is expected to find its application in various fields including drug delivery system (DDS), coating technology, parts for micromachine, etc., because of its quick environmental response. Monodisperse microgels of $\mu \mathrm{m}$ or sub- $\mu \mathrm{m}$ order in diameter can be prepared by emulsion polymerization or precipitation polymerization. The latter polymerization makes use of the precipitation of polymer resulting from homogeneous monomer solution. Recently it was found that monodisperse microgel could be prepared by precipitation polymerization from acrylamide (AAm) and methylenebisacrylamide (MBAAm) in alcohol adding a proper amount of methacrylic acid (MAc) without using any stabilizer. ${ }^{1}$ The particle size can be controlled in some extent by adjusting the composition of solvent, monomers and initiator. ${ }^{2-4}$ In order to investigate the microgel formation mechanism in this system, the particle size and the swelling degree were measured at various conversions. The internal structure of microgels was observed at several tens to hundreds $\AA$ by means of small-angle X-ray scattering (SAXS).

\section{EXPERIMENTAL}

\section{Materials}

AAm (first-grade reagent purchased from Wako Pure Chemicals Co., Ltd.) was recrystallized. MBAAm (special-grade reagent from Wako Pure Chemicals Co., Ltd.) was used without further purification. MAc (specialgrade reagent from Wako Pure Chemicals Co., Ltd.) was distilled under a reduced pressure $\left(40^{\circ} \mathrm{C}, 3 \mathrm{mmHg}\right)$ to remove polymerization inhibitor and oligomer. The chloroform solution of azobisisobutyronitrile (an initiator, AIBN) (special-grade reagent from Wako Pure Chemicals Co., Ltd.) was filtered and AIBN was precipitated by adding purified methanol. Pure AIBN powder

\footnotetext{
† To whom correspondence should be addressed.
}

was obtained by filtration and drying under a reduced pressure. Isopropyl alcohol (special-grade reagent from Wako Pure Chemicals Co., Ltd.) was used as received.

\section{Polymerization}

Weighed monomers and alcohol were put into a $100 \mathrm{ml}$ round-bottomed flask with four necks carrying a stirring equipment, a nitrogen-inducing tube, a condenser and a serum rubber. Nitrogen gas was blown into the solution for one hour at $60^{\circ} \mathrm{C}$ with stirring at $200 \mathrm{rpm}$ and then AIBN dissolved in alcohol was injected to it to initiate polymerization.

Resulted polymer latex was refined by centrifugation. The latex dispersion was centrifuged at $12000 \mathrm{rpm}$ for 20 minutes and the precipitate was re-dispersed in water or other solvents after removing a top clear layer by decantation. This process was repeated five times.

\section{Measurements}

Total conversion was evaluated by gravimetry. The size and shape of particles were observed by scanning electron microscopy (SEM) (JEOL JSM-5200 Japan Electronics Co., Ltd.) after $\mathrm{Pd}-\mathrm{Au}$ vacuum evaporation onto dried polymer. The hydrodynamic size of particles was measured by photon correlation spectroscopy (PCS) equipment composed of LPA-3000 (Laser Particle Analyzer) and LPA-3100 (Photon Correlator) (Ohtsuka Electronics Co., Ltd.).

SAXS measurements were carried out with a smallangle X-ray equipment for solution (SAXES) installed at BL-10C in the Photon Factory, Tsukuba, Japan. An incident X-ray from synchrotron radiation was monochromatized to the wavelength of $1.49 \AA$ and focused to the focal point with a bent focusing mirror. The scattered $\mathrm{X}$-rays were detected by a one-dimensional position sensitive proportional counter (PSPC) positioned at the distance about 1 meter from the sample holder. A flat sample cell of $0.1 \mathrm{~cm}$ in path-length is made of stainless 
Table I. Apparance of microspheres prepared by precipitation copolymerization of AAm, MBAAm, and MAc in isopropyl alcohol ${ }^{\mathrm{a}}$

\begin{tabular}{|c|c|c|c|c|c|c|}
\hline Sample & $\mathrm{AAm} / \mathrm{g}$ & $\mathrm{MBAAm} / \mathrm{g}$ & $\mathrm{MAc} / \mathrm{g}$ & IPA $/ \mathrm{g}$ & $\begin{array}{l}\text { Dispersion } \\
\text { condition }\end{array}$ & Monodispersity \\
\hline AAm & 5 & 0 & 0 & 45 & Bad & Bad \\
\hline $\mathrm{AAm} / \mathrm{MB}$ & 4 & 1 & 0 & 45 & Bad & $\mathrm{Bad}$ \\
\hline $\mathrm{AAm} / \mathrm{MB} / \mathrm{MAc}$ & 3 & 1 & 1 & 45 & Good & Good \\
\hline $\mathrm{MB} / \mathrm{MAc}$ & 0 & 2.5 & 1 & 46.5 & $\mathrm{Bad}$ & Good \\
\hline $\mathrm{AAm} / \mathrm{MAc}$ & 4.4 & 0 & 1.3 & 47.2 & Good & Good \\
\hline
\end{tabular}

${ }^{\mathrm{a}} \mathrm{AIBN}, 0.025 \mathrm{~g}$.

steel with quartz plates of $10 \mu \mathrm{m}$ in thickness for a window. Excess scattering from a microgel was evaluated by subtracting the scattering of solvent from that of microgel dispersion.

\section{RESULTS AND DISCUSSION}

Precipitation polymerization from AAm alone in alcohol yielded bulky aggregates of $100-200 \mu \mathrm{m}$ composed of minute particles. As reported in previous papers, ${ }^{1,2}$ introducing MBAAm as a crosslinker and $\mathrm{MAc}$ as an ionic monomer made it possible to prepare a monodisperse hydrogel microsphere of sub- $\mu \mathrm{m}$ size. We examined the preparation from various monomer recipes indicated in Table $I$. It was confirmed that the polymerization containing MAc could afford a good monodispersity of particles and the stability of particle dispersion. The polydispersity with regard to the particle size was evaluated from the observed SEM views, when the microsphere particles revealed a sharp size distribution of $D_{w} / D_{n}=1.01$ with $D_{w}$ being a weight average diameter and $D_{n}$ a number average diameter. MAc is thought to be a key factor to produce fine monodisperse hydrogel microspheres, and to function as a dispersing agent.

\section{Characterization of Microgel Structure in Polymerization Process}

To elucidate the mechanism of the microsphere formation from AAm, MBAAm, and MAc, we observed a series of particles at different conversions by various methods. Here the precipitation polymerization was undertaken from the mixture of $\mathrm{AAm} / \mathrm{MBAAm} / \mathrm{MAc}=$ $0.72 / 0.08 / 0.20$ in terms of molar fraction in $240 \mathrm{ml}$ isopropanol. Figure 1 shows the particle growth expressed in terms of the diameter in dried state measured by SEM and the hydrodynamic diameter by PCS as a function of conversion. Both diameters increase as the reaction proceeds, where the diameter in dried state was found to increase in proportion to (conversion) ${ }^{1 / 3}$ as indicated in Figure 2. This result indicates that the number of particles is determined at the beginning of reaction and then remains constant. In the course of reaction, radical oligomers are incorporated into already existing particles and form no new nuclei to produce polymer particles.

The degree of swelling was calculated as the ratio of two diameters estimated above at each conversion (Figure 3). It had a minimum at about $35 \%$ in conversion when the crosslinking density is supporsed to be the highest. The crosslinking of MBAAm proceeds as a dominant reaction in the begining up to the conver-

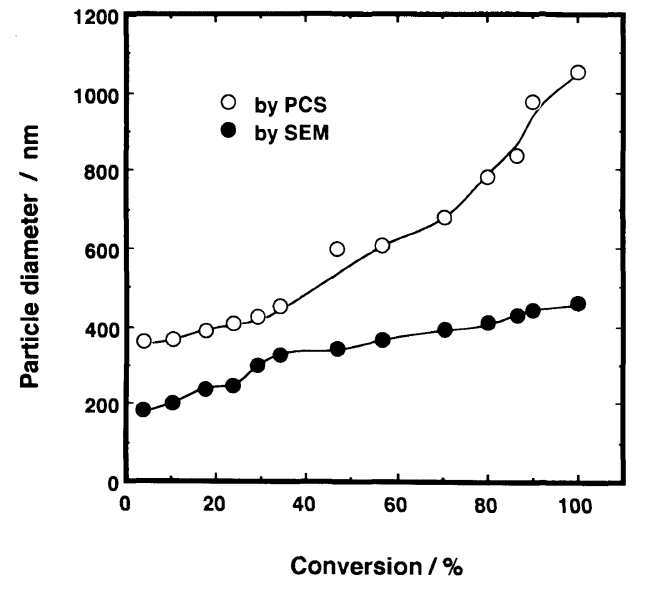

Figure 1. Dependence of the diameter of terpolymer particles on conversion in the precipitation copolymerization of AAm, MBAAm, and MAc in isopropanol. Here the total monomer concentration was $1.3 \mathrm{moll}^{-1}$, with $\mathrm{AAm} / \mathrm{MBAAm} / \mathrm{MAc}=0.72 / 0.08 / 0.20$ in terms of molar fraction in $240 \mathrm{ml}$ isopropanol containing $0.025 \mathrm{~g}$ AIBN.

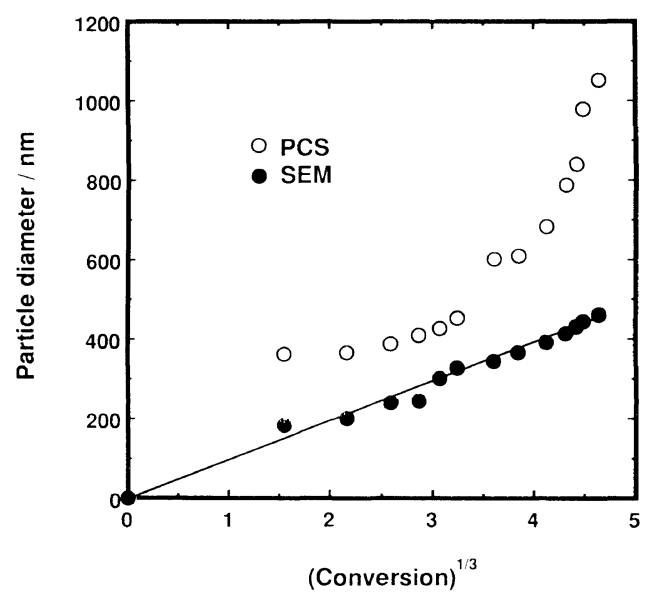

Figure 2. Relation between diameter of terpolymer particles and (conversion) $)^{1 / 3}$.

sion around $35 \%$ and in consequence the swelling degree is suppressed. Then AAm reacts into linear polymers incorporated in network, so that the crosslinking density is reduced to increase the degree of swelling.

The amount of carboxyl groups was measured at different conversions by conductometric titration (Figure 4). The carboxyl content was evaluated as $2.68 \mathrm{mmol} \mathrm{g}^{-1}$ at the end of reaction, which was close to the theoretical value $2.48 \mathrm{mmol} \mathrm{g}^{-1}$. Higher values were recorded at early stage, where MAc was consumed at higher ratio. Thus MAc is considered to function as a dispersion stabilizer in the course of microsphere formation.

Polym. J., Vol. 28, No. 4, 1996 


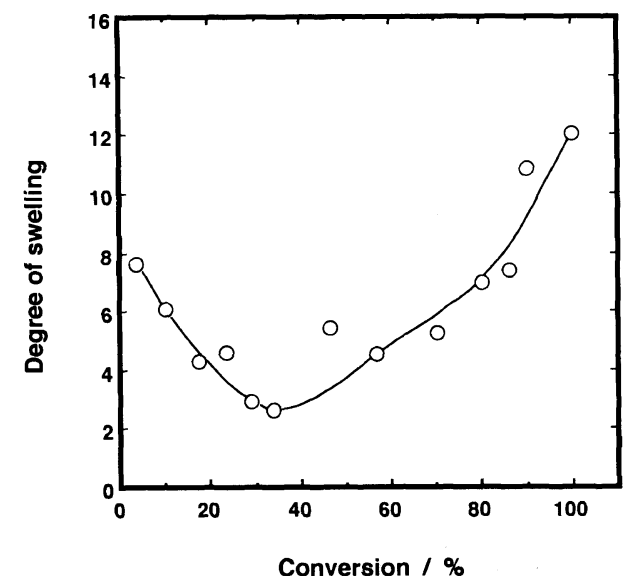

Figure 3. Dependence of the degree of swelling on conversion.

$$
\text { Degree of swelling }=\frac{(\text { Hydrodynamic diameter of particles })^{3}}{{\text { (Diameter of dried particles })^{3}}^{3}}
$$

Hydrodynamic diameter of particles: meaeured by PCS. Diameter of dried particles: observed by SEM.

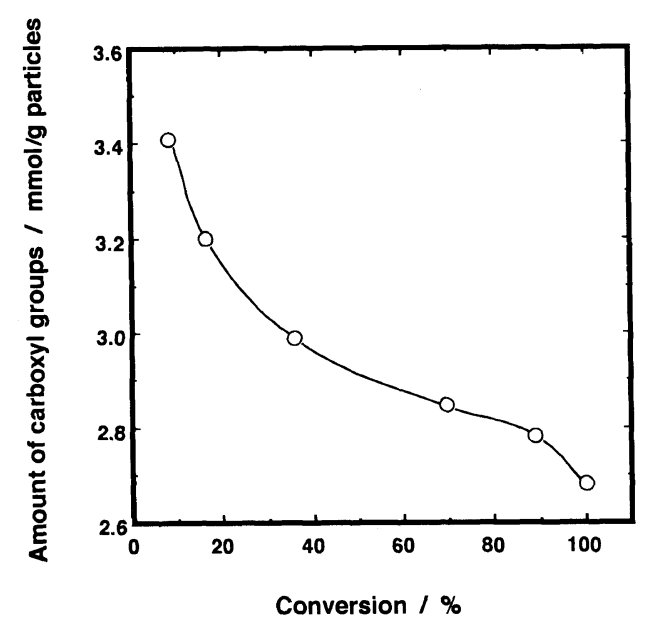

Figure 4. Dependence of the amount of carboxyl groups on conversion.

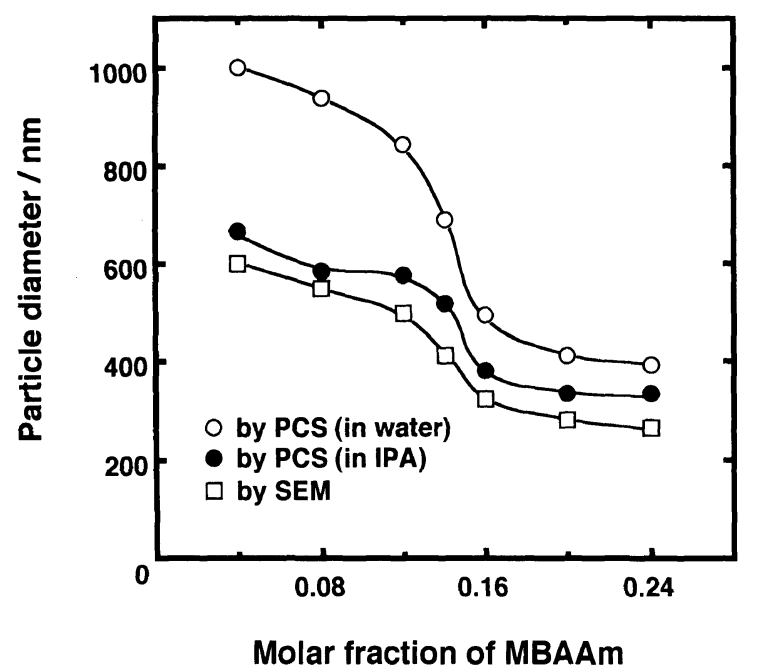

Figure 5. Dependence of the diameter on the molar fraction of MBAAm. (Polymerization condition: a total monomer concentration $1.3 \mathrm{moll}^{-1}$; a dispersant $60 \mathrm{ml}$ isopropanol; AIBN $0.025 \mathrm{~g}$.)

\section{Microspheres Polymerized in Various Conditions}

Microspheres were prepared with the conditions varying monomer compositions, monomer concentrations

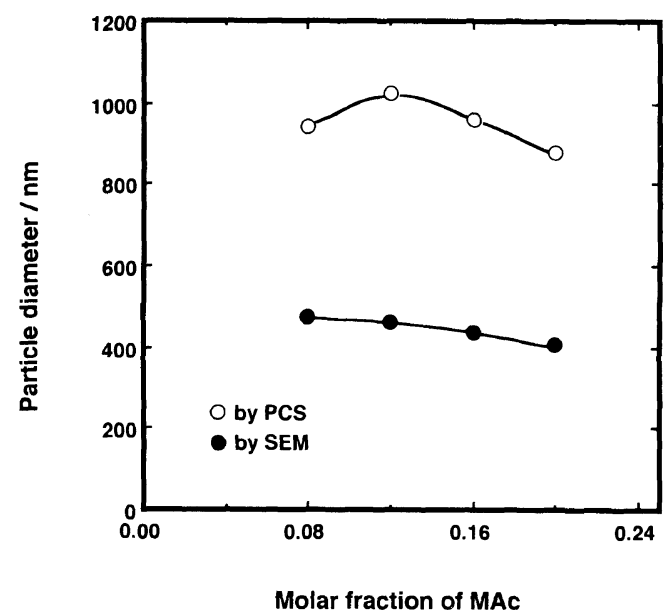

Figure 6. Dependence of the diameter on the molar fraction of MAc. (Polymerization condition: MBAAm concentration was fixed to 0.08 in terms of molar fraction, a total monomer concentration $1.3 \mathrm{moll}^{-1}$; a dispersant $60 \mathrm{ml}$ isopropanol; AIBN $0.025 \mathrm{~g}$.)

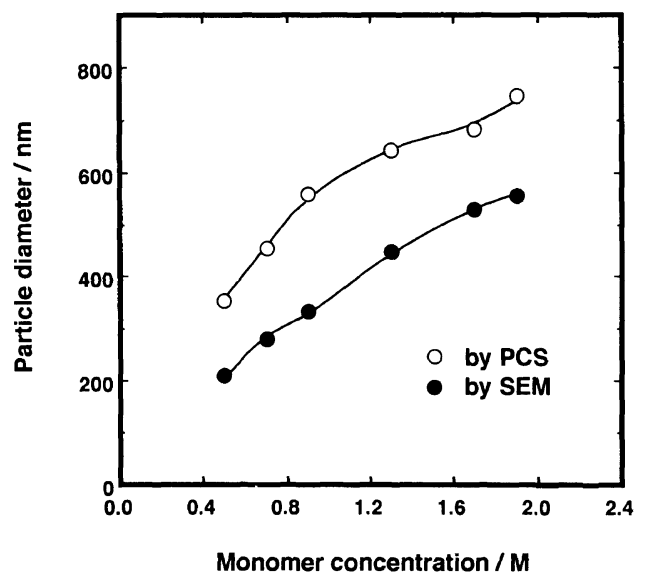

Figure 7. Dependence of the diameter on the total monomer concentration. (Polymerization condition: a molar fraction of monomers $\mathrm{AAm} / \mathrm{MBAAm} / \mathrm{MAc}=0.68 / 0.12 / 0.20$; a dispersant $60 \mathrm{ml}$ isopropanol.)

Table II. Conversion and hydrodynamic diameter of microspheres as a function of the amount of AIBN ${ }^{a}$

\begin{tabular}{ccc}
\hline AIBN/g & Conversion/\% & $\begin{array}{c}\text { Hydrodynamic } \\
\text { diameter/nm }\end{array}$ \\
\hline 0.010 & 10.0 & 313.7 \\
0.015 & 54.5 & 385.2 \\
0.025 & ca. 100.0 & 628.6 \\
0.050 & ca. 100.0 & 521.5 \\
0.075 & ca. 100.0 & 483.6 \\
\hline
\end{tabular}

a AAm/MBAAm/MAc/IPA (g)=3/1/1/45.0; polymerization time, 22 hours.

and initiator concentrations. The microsphere diameter was found to decrease as the MBAAm concentration increased from 0.04 to 0.24 in molar fraction in the starting reaction mixture where the MAc concentration was fixed to 0.20 (Figure 5). The degree of swelling in water showed the same tendency as the diameter. The high content of MBAAm is thought to result highcrosslinked, rigid and small-sized particles.

The MAc concentration hardly affects the size of resulted particles in the examined range (Figure 6). On the contrary, when the monomer concentration was 
increased, the diameter of resulted particles became proportionally larger (Figure 7). Here the solvent containing higher concentration of monomers is a better solvent for polymer particles, so that the precipitation of polymer

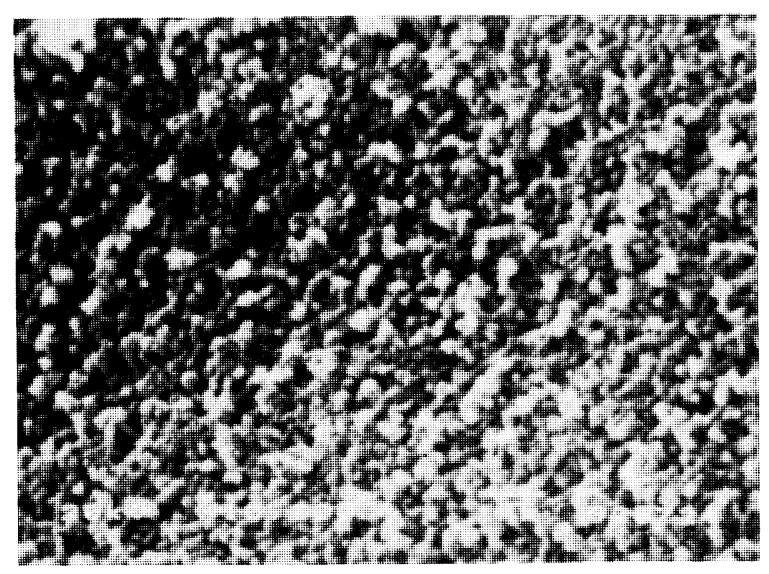

\section{$8.23 \%(20 \mathrm{~min})$}

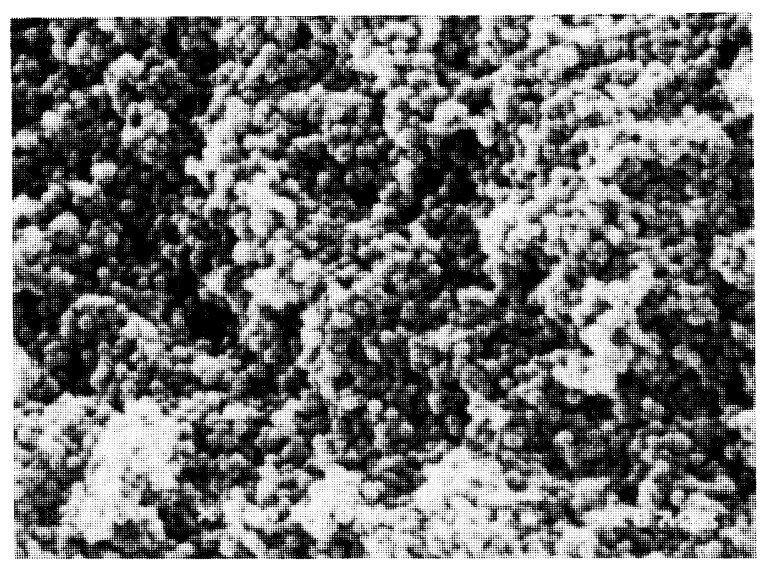

\section{$29.9 \%(30 \mathrm{~min})$}

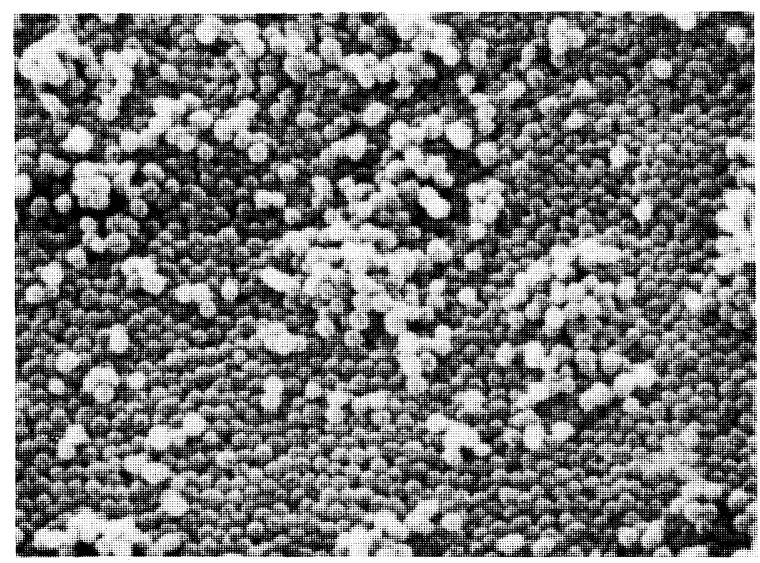

$38.2 \%(1 \mathrm{~h})$ nuclei may be delayed to reduce the number of resulted particles and increase the size.

An initiator is also an influential factor to determine the particle size. Table II shows that the reaction cannot

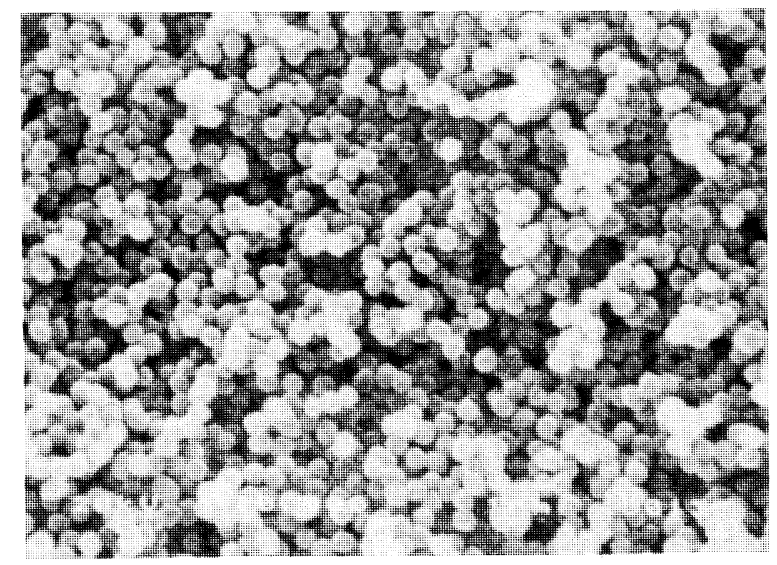

$83.1 \%(3 \mathrm{~h})$

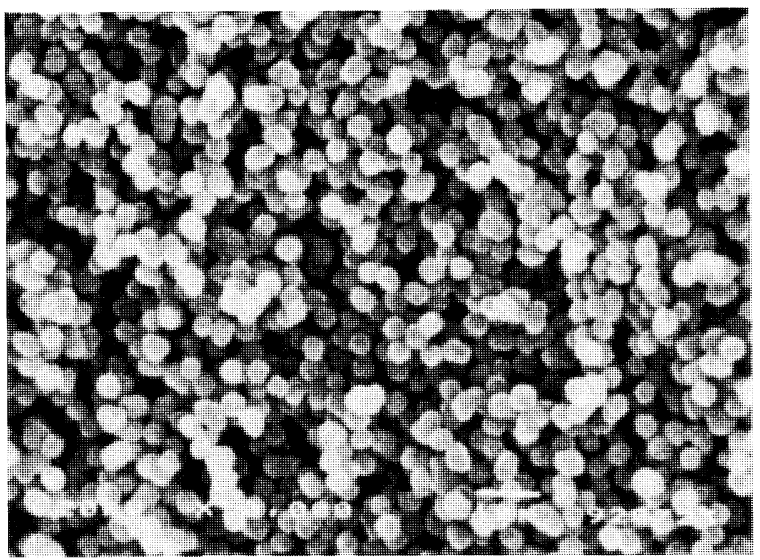

$90.2 \%(5 \mathrm{~h})$

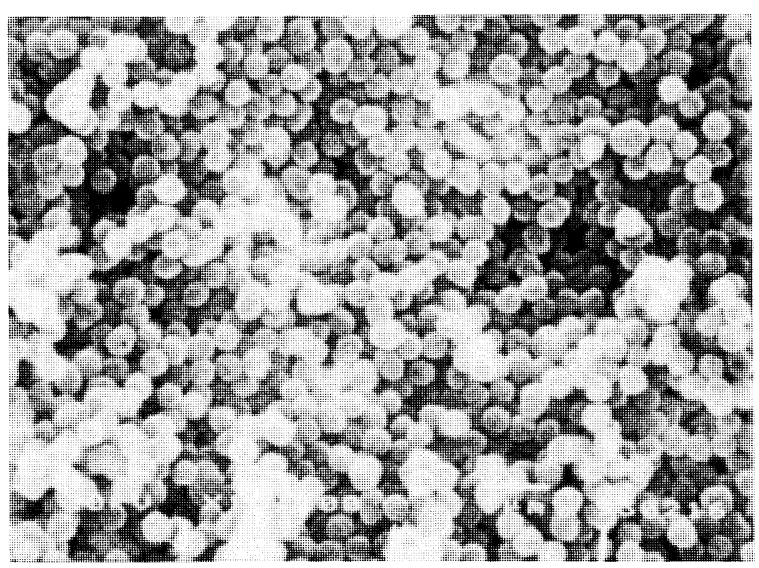

$100.0 \%(22 \mathrm{~h})$

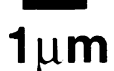

Figure 8. SEM views of terpolymer particles at various conversions for SAXS measurements. Polymerization was carried out with AAm/ MBAAm $/$ MAc $=0.72 / 0.08 / 0.02$ (molar fraction) in $57 \mathrm{ml}$ isopropanol. A total monomer concentration was $1.1 \mathrm{~mol} \mathrm{l}^{-1}$. The conversions and polymerization times are indicated in the figure. 
be completed with the small amount of AIBN but a further increase of AIBN reduces the size of resulted microspheres.

Internal Structure Observed by Small-Angle X-Ray Scattering

Small-angle X-ray scattering (SAXS) allows us to analyze the structural characteristics of a scattering body in order of 5 to $500 \AA$, although the resolution depends heavily on the instrumental set-up. Thus the SAXS profile from microgels ${ }^{5,6}$ is expected to reflect the local structure of microgel (i.e., internal structure), but not to reveal the scattering from an entire spherical shape of a few hundreds nm's. In this section the SAXS was observed from a series of the microgel samples newly prepared for this purpose at various conversions. These SAXS samples were prepared with monomer recipe, AAm/ MBAAm $/ \mathrm{MAc}=0.70 / 0.11 / 0.19$ in terms of molar fraction, and the solutions for SAXS were adjusted to the concentration summarized in Table III by dissolving the refined microgels into an appropriate amount of water.
The particle shape dried from alcohol suspensions was observed by SEM as shown in Figure 8. The hydrodynamic diameters were evaluated by PCS for the microgel samples at various conversions as summarized in Table III. The SAXS profiles from corresponding samples (the dependence of scattering intensities $I(q)$ on the

Table III. Characteristics of the microsphere samples subjected to SAXS measurements ${ }^{\text {a }}$

\begin{tabular}{cccc}
\hline $\begin{array}{c}\text { Polymerization } \\
\text { time }\end{array}$ & Conversion/\% & $\begin{array}{c}\text { Hydrodynamic } \\
\text { diameter/nm }\end{array}$ & $\begin{array}{c}\text { Concentration } \\
\text { of samples } \\
\text { for SAXS } / \%\end{array}$ \\
\hline 20 minutes & 8.23 & 285.0 & 0.57 \\
30 minutes & 29.9 & 341.9 & 1.0 \\
1 hour & 38.2 & 412.0 & 1.0 \\
2 hours & 64.8 & 582.0 & 1.0 \\
3 hours & 83.1 & 700.0 & 1.0 \\
5 hours & 90.2 & 785.0 & 1.0 \\
22 hours & 100.0 & 799.0 & 1.0 \\
\hline
\end{tabular}

a Preparation condition: AAm/MBAAm/MAc/AIBN/IPA = 3/1/1/ $0.025 / 45(\mathrm{~g})$.

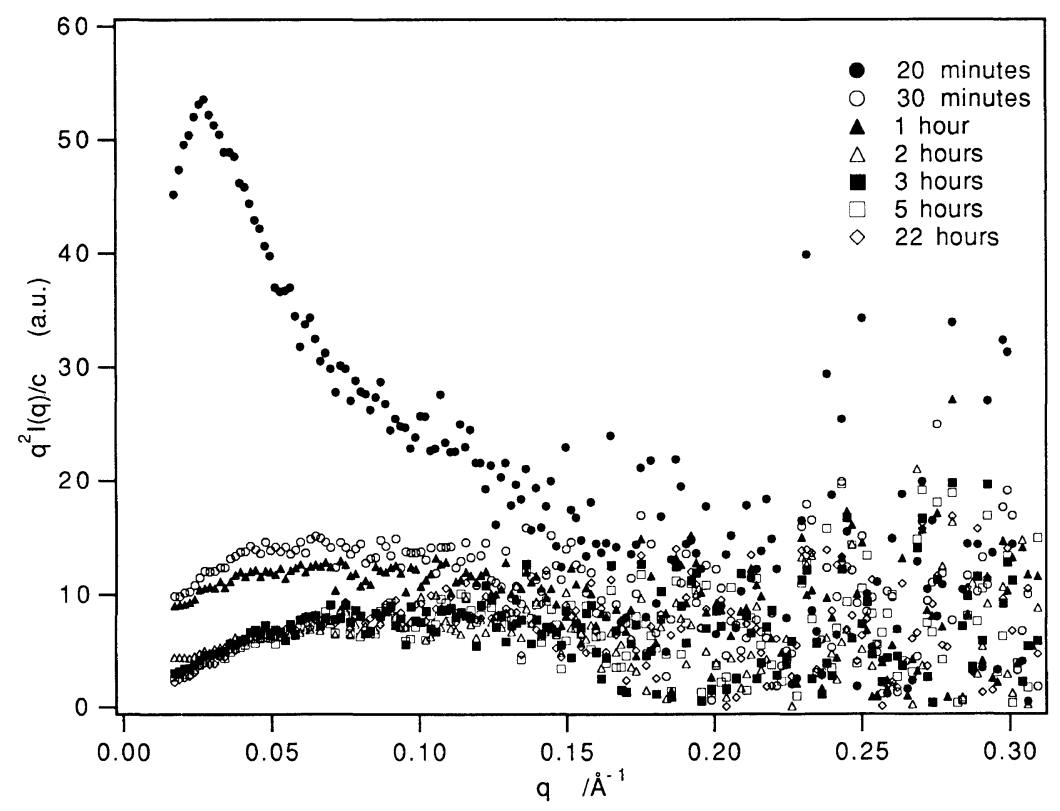

Figure 9. Kratky plot for SAXS from microgels as a function of reaction time.

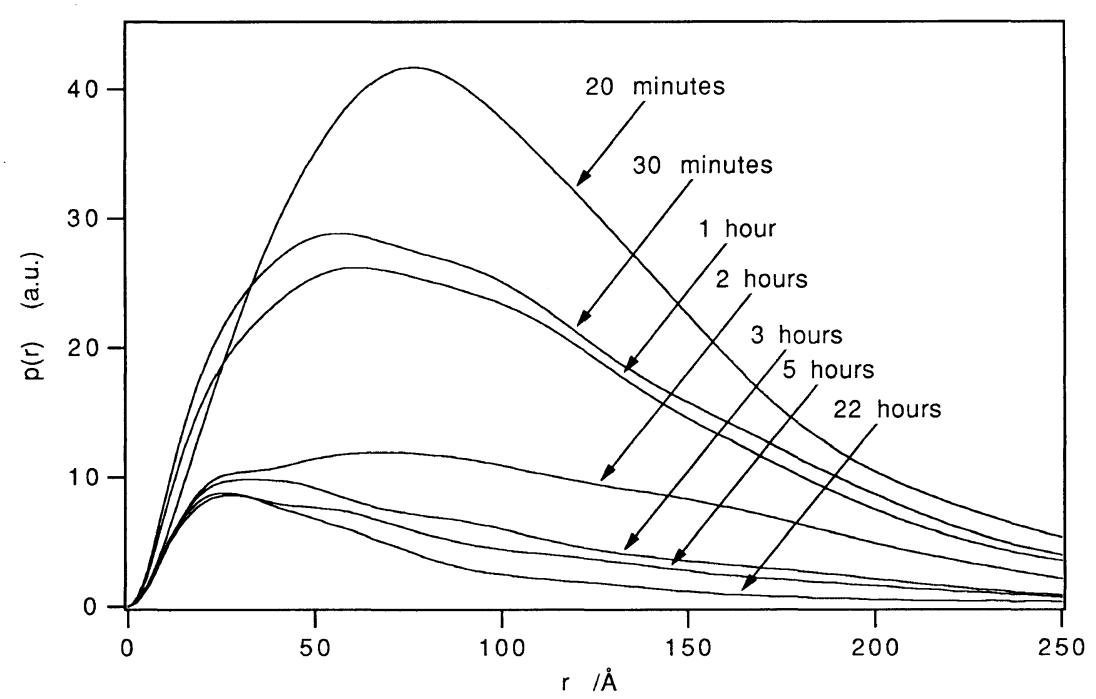

Figure 10. Distance distribution function $p(r)$ of microgels as a function of reaction time. 
magnitude of scattering vector $q=4 \pi / \lambda \sin (\theta / 2)$ where $\lambda$ and $\theta$ are the wavelength of an incident $\mathrm{X}$-ray and a scattering angle, respectively) are shown in Figure 9 in terms of Kratky plots $\left(q^{2} I(q) v s . q\right)$. The SAXS profile from the sample prepared at the reaction time 20 minutes, exhibited a maximum at $q=0.028 \AA^{-1}$. The appearance of a maximum in Kratky plot is considered to be due to a sharp decay of density correlation around a certain correlation length. Scince scattering profile at higher $q$ region maintains a characteristic of flexible chain structure, the profile is thought to be the scattering from a star-shaped polymer composed of linear polymers radiating from a nucleus. ${ }^{7}$

As polymerization proceeds, the maximum disappears and the scattering intensity decreases. SAXS profiles were inversely Fourier-transformed to the distance distribution function $p(r)$ (Figure 10), which describes a space correlation of electron density at a distance $r$
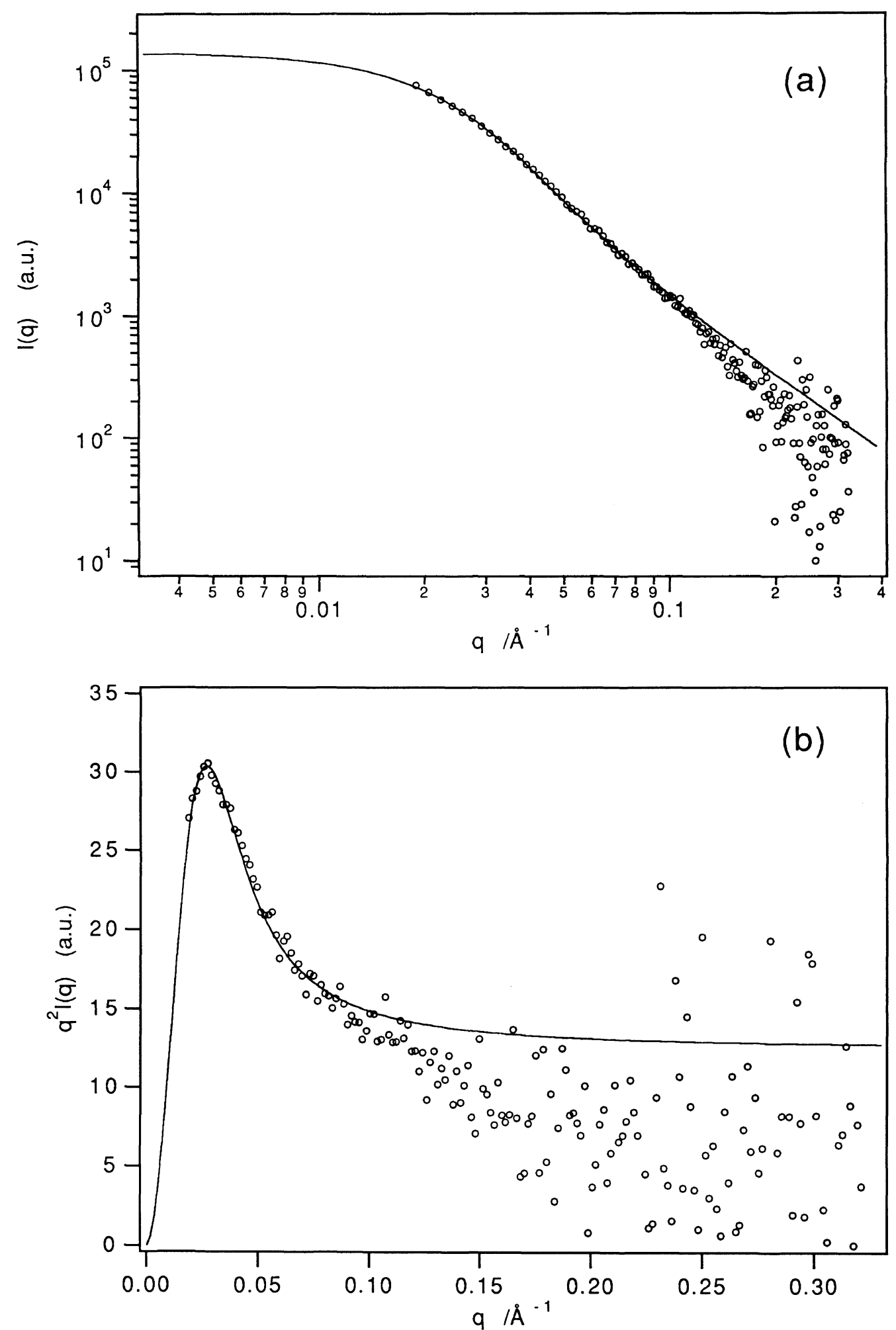

Figure 11. SAXS profile at 20 minutes reaction (open circle) and a simulated profile (solid line) assuming a star-shaped Gaussian chain with $f=10.7$ ( $f$, the number of rays). (a) the double logarithmic plot, (b) the Kratky plot. 
between any two points in the scattering body, according to the equation;

$$
p(r)=(2 \pi)^{2} \int_{0}^{\infty} I(q) \cdot(q r) \cdot \sin (q r) \mathrm{d} q
$$

$p(r)$ in the sample of 20 minutes microgel exhibits a peak around $r=80 \AA$. As reaction proceeds, a peak position shifts to a shorter distance, and is finally settled at $r \sim$ $25 \AA$ after 5 hours.

The profile of Kratky plot suggests the formation of star-shaped polymers at early polymerization stage. Assuming the Gaussian chain distribution, the scattering profiles were simulated by a star-shaped chain with $c a .12$ branches of equal length (Figure 11). The deviation at high $q$ 's is probably due to the local chain conformation different from a Gaussian chain. Here the radius of gyration was found to be $76.4 \AA$ which corresponds to $44.1 \AA$ in terms of the correlation length $\xi$. As the reaction proceeds, the clusters grow partly by coupling starshaped polymers and the network structure (eventually gel) is formed with remaining double bonds of MBAAm in the clusters. The coupling seems to take place after 30 minutes reaction, where the profile of Kratky plot reveals a typical scattering from semi-flexible linear chains. Network formation cancels the structural characteristics of star-shaped polymers, and mainly the SAXS is considered to reflect the structure of the network chains.

Since the star-shaped nuclei are coupled to form microspheres, each microsphere is assumed to be composed of domains specified by the correlation length $\xi$. Each domain contributes independently to scattering, and no interference will be observed at $q \geq \xi^{-1}$. A density fluc- tuation inside a domain is assumed to be given in terms of the density correlation function for a simple fractal

$$
\gamma(r) \sim(\xi / r)^{3-D} \exp (-r / \xi)
$$

where $D$ is a fractal dimension. By Fourier-transformation of this correlation function, the scattering function $I(q)$ is approximately given as ${ }^{8,9}$

$$
I(q) \sim \frac{c \xi^{3}}{\left(1+\xi^{2} q^{2}(D+1) / 3\right)^{D / 2}}
$$

For example, eq 3 yields the Ornstein-Zernike type scattering function ${ }^{10}$ when $D=2$ (equivalent to a Gaussian chain).

The correlation length $\xi$ and the fractal dimension $D$ were evaluated by fitting eq 3 to the SAXS profiles from microgel as summarized in Table IV. The calculated profiles revealed a good agreement with SAXS from microgels as seen in Figure 12. The correlation length $\xi$ takes the maximum value of $54 \AA$ at 1 hour in reaction time when reached to the gel point of microspheres and decreases to $\xi \sim 30 \AA$ as reaction proceeds, while the

Table IV. Correlation length and fractal dimension evaluated by curve fitting according to eq 3

\begin{tabular}{ccc}
\hline Sample & Correlation length $\xi / \AA$ & Fractal dimension $D$ \\
\hline 30 minutes & 45.8 & 2.27 \\
1 hour & 53.8 & 2.21 \\
2 hours & 47.0 & 2.03 \\
3 hours & 33.6 & 2.11 \\
5 hours & 33.8 & 2.00 \\
22 hours & 31.5 & 1.96 \\
\hline
\end{tabular}

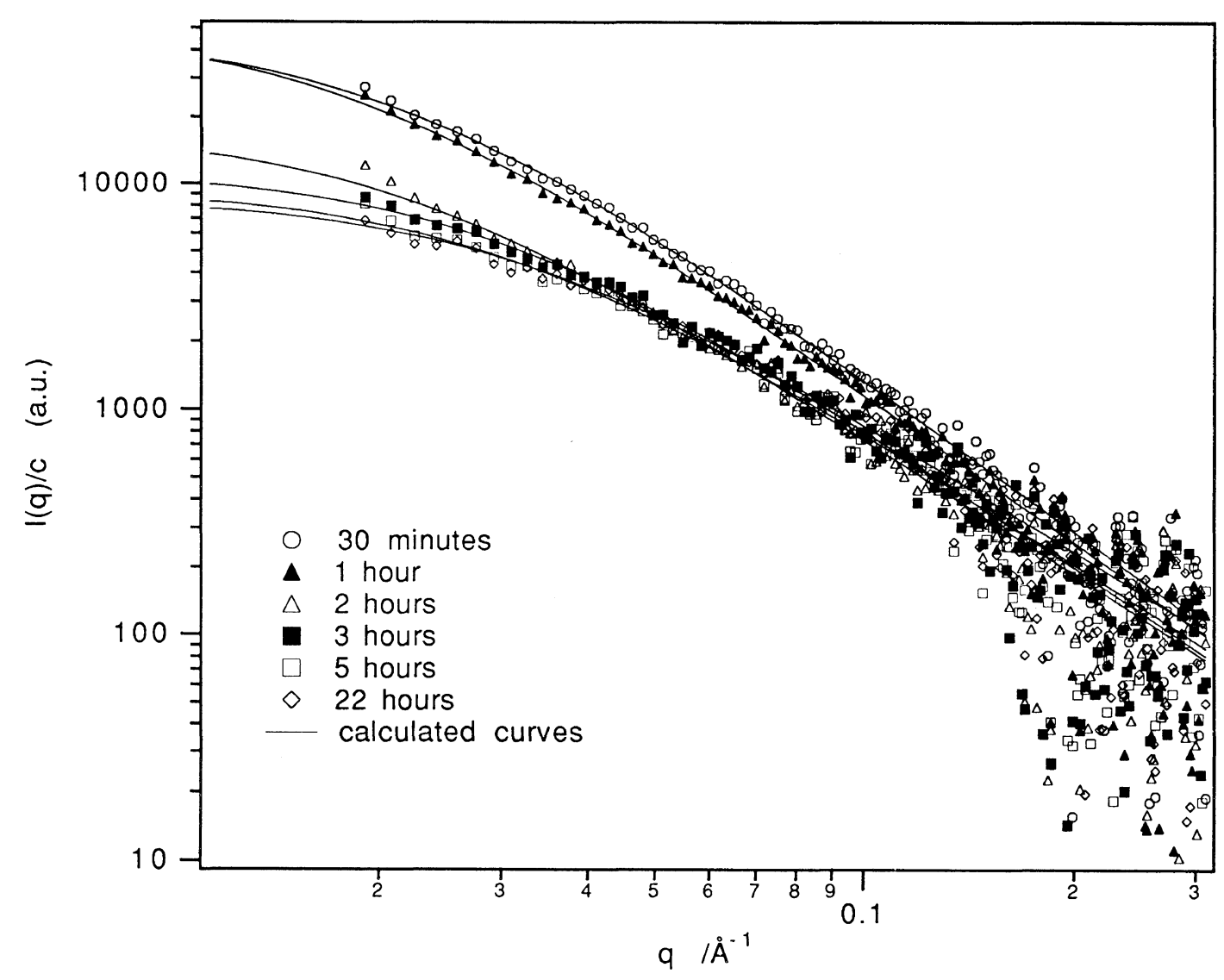

Figure 12. Double-logarithmic plots of SAXS and the fitted curve according to eq 3 at various reaction times. 
fractal dimension $D$ monotonously decreases from $\sim 2.3$ to $\sim 2.0$. Since the SAXS reveales the characteristic profile of star-shaped structure prior to the $30 \%$ conversion, the crosslinking reaction is speculated to proceed in two steps of (1) the formation of star-shaped nuclei and the subsequent aggregation of the nuclei, which are completed around the $20-25 \%$ conversion and (2) the coupling of nuclei to build network and the subsequent micro-gelation around the $40 \%$ conversion. The further reaction seems to incoporate remaining AAm and form the less-crosslinked surface layer on microgels. The crosslinking reaction still proceeds in the microgels. The network chains become less flexible by crosslinking, so that the fractal dimension $D$ decreases.

\section{CONCLUSION}

The following conclusion can be summarized for the formation of microgels prepared from AAm, MBAAm, and MAc. MAc was found to be essential as a dispersion stabilizer to yield monodisperse microspheres. The microsphere particles are formed predominantly by crosslinking agents at early stage reaction, where star-shaped nuclei are composed and coupled to constitute network. At this stage the swelling degree decreases due to the increase of crosslinking density up to a gel point. The particle size was found to depend on the monomer composition, the amount of initiator, and the total monomer concentration. Then the further growth of the microspheres follows by incorporating remaining AAm onto the microsphere surface.

Acknowledgments. The work was performed under the approval of Photon Factory Advisory Committee (Proposal No. 92-G273).

\section{REFERENCES}

1. H. Kawaguchi, Y. Yamada, S. Kataoka, Y. Morita, and Y. Ohtsuka, Polym. J., 23, 955 (1991).

2. H. Kawaguchi, K. Fujimoto, M. Saito, T. Kawasaki, and Y. Ohtsuka, Polym. Inter., 30, 225 (1993).

3. M. Saito, Diplom. Thesis, Keio University (1992).

4. Y. Kamijo, Diplom. Thesis, Keio University (1994).

5. D. Nerger, K. Kajiwara, H. Urakawa, and Y. Hiragi, Bull. Inst. Chem. Res., Kyoto Univ., 67, No. 2, 97 (1987).

6. M. A. Winnik, B. Williamson, and T. P. Russell, Macromolecules, 20, 899 (1987).

7. W. Burchard, Adv. Polym. Sci., 48, 68 (1983).

8. K. Kajiwara, S. Kojiya, M. Shibayama, and H. Urakawa, in "Polymer Gels; fundamentals and Biomedical Applications," D. DeRossi, K. Kajiwara, Y. Osada, and A. Yamauchi, Ed., Plenum Press, New York, N.Y., 1991, p 3.

9. M. Shibayama, H. Kurokawa, S. Nomura, M. Muthukumar, R. S. Stein, and S. Roy, Polymer, 33, 2883 (1992).

10. P. G. de Genne, "Scaling Concepts in Polymer Physics," Cornell University Press, Ithaca, New York, 1979. 